\title{
CrimRxiv
}

\section{Pathways to Recovery and Desistance: The Role of the Social Contagion of Hope}

\section{David Best}

Published on: Jul 01, 2019

DOI: $10.21428 / c b 6 a b 371.0716185 b$

License: Creative Commons Attribution 4.0 International License (CC-BY 4.0). 
